\title{
Rusia: dos herencias
}

\author{
Rusia: Two Inheritances
}

José Ernesto Rangel Delgado ${ }^{1}$

\section{Resumen:}

En este artículo se presentan dos momentos en la historia de Rusia que cambiaron el mundo: la Revolución de Octubre, en contra de una Monarquía Imperial y el posicionamiento de un poder soviético que logró transitar hacia el Capitalismo, sin el derrame de una sola gota de sangre. Ambos eventos se suscitaron como hitos para el surgimiento de un mundo bipolar y, posteriormente, para su desaparición.

Palabras clave: Rusia, Revolución, Capitalismo.

\begin{abstract}
:
This article presents two moments in the history of Russia that changed the world: the October Revolution, against an Imperial Monarchy, and the positioning of a Soviet power that managed to move towards Capitalism, without the spill of a single drop of blood. Both events were raised as milestones for the emergence of a bipolar world and, subsequently, for its demise.
\end{abstract}

\section{Introducción}

Dos grandes momentos de naturaleza rusa cambiaron la historia. El primero fue la Revolución de Octubre, en 1917, con el que se abrió el camino a una era de esperanzas a favor de un humanismo lastimado por el desarrollo industrial capitalista acelerado. El segundo fue el pronunciamiento de un mundo "sin muro", en 1989, que fue resultado de una intensa lucha por establecer condiciones de bienestar en pro de la democracia

\footnotetext{
${ }^{1}$ Profesor investigador de la Facultad de Economía y del Centro de Estudios e Investigaciones sobre la Cuenca del Pacífico de la Universidad de Colima.
} 
occidental y que enarboló las libertades políticas de los ciudadanos, a la par de un consumo marcado por el derecho de elegir.

Sin embargo, es necesario considerar las aristas de estos eventos: el primer cambio mermó en la falta de condiciones internas de igualdad, propiciada por la Monarquía en decadencia; el segundo desencadenó un panorama económico-social inestable. Fue posteriormente, cuando se diseñaron planes para solventar estas problemáticas, que ambos fenómenos desprendieron de su naturaleza un espíritu de libertad, que se esparció en los pueblos.

\section{La Revolución de 1917}

La Revolución Rusa de 1917, iniciada en febrero y posteriormente encumbrada en octubre, en sintonía con los trágicos estragos de la Guerra civil, dio lugar a nuevas estrategias de desarrollo que no desconocieron las formaciones sociales del Capitalismo. Tal fue el caso de la Nueva Política Económica (NEP); aunque en las etapas siguientes, la ya formada Unión Soviética ponderó la consolidación de un estado unificado, estableciendo las bases para un proceso de industrialización acelerado que generó los principios de su desarrollo.

En este marco, la Revolución de Octubre fue un estandarte para que otros países pugnaran por cambios sustantivos en su orden. Por ejemplo, México proclamó su tercera Constitución Política. Además, fue un parteaguas para la transformación de sus estructuras internas; cabe resaltar el reconocimiento de Valentina Kolontái como la primera mujer con un cargo diplomático en el mundo -designada por la Unión de Repúblicas Socialistas Soviéticas (URSS) como embajadora en México-; también es importante subrayar la aceptación de direcciones con una marcada orientación socialista, que, según la Tesis del Capitalismo de Estado (de Lapshov, 1990) constatan un fuerte intervencionismo estatal proveniente de un sólo partido.

Puede decirse que uno de los principales aportes de este nuevo modelo fue el impulso al crecimiento económico acelerado, basado en una industrialización nacional sólida. Este modelo se extendió más allá de las fronteras de la URSS y llegó hasta una zona de Europa, considerada desde entonces del Este; pero también se extendió hasta las tierras del lejano Oriente. 


\subsection{Marco cronológico}

La primera Revolución de 1917 puede ser considerada como el antecedente más inmediato del surgimiento de la URSS. Según Danilov (2012), con este movimiento se registraron cambios urgentes: la liquidación de la propiedad privada, el establecimiento de una legislación industrial progresista, la regulación de una jornada laboral de 8 horas, la limitación al poder del Zar y la resolución del problema de las nacionalidades, ocasionado por el carácter multinacional de Rusia. Estas mejoras impactaron positivamente en la sociedad, que encontró alicientes para problemáticas de antaño, como insuficiencia en la producción de alimentos, el mal funcionamiento del sistema de ferrocarriles y la falta de fuentes de energía para soportar el fuerte invierno.

Posteriormente, la Revolución de Octubre demarcó otros antecedentes para el surgimiento de la URSS, principalmente relacionados con la democratización de la sociedad y la libertad obrera y campesina -sometidas por la monarquía y por los imperios extramuros-; de esta forma, instauró una forma de gobierno que centralizó el poder y la nacionalización de las finanzas. Entonces se lograron las elecciones más democráticas de la historia, que lograron la proclamación de la "Declaración de los derechos de los trabajadores y del pueblo explotado", sobre la cual se sentaban las bases de los primeros decretos del poder soviético; estos principios consideraban la eliminación de las clases sociales, la separación Iglesia-Estado/Iglesia-Educación, así como la igualdad jurídica entre las mujeres y los hombres.

En tanto, el Congreso de los Sóviets fue nombrado el máximo órgano legal de este nuevo orden. Por otra parte, en diciembre del 1917 fue constituida la Comisión Extraordinaria de todas las Rusias, cuya finalidad era resistirse a los movimientos contrarrevolucionarios y al sabotaje.

Más tarde, en enero de 1918, se constituyó la Armada Soviética. En este ejército, los sóviets regionales se ramificaron en dumas urbanas y cada una de las asambleas locales y provinciales, elegidas por la nobleza en la era zarista, tomó el poder en sus manos; con la diferencia de que ahora eran dirigidos por miembros del Partido Bolchevique.

En ese año, Rusia tendría que enfrentarse a una Guerra civil originada por el carácter impopular del gobierno. Este conflicto se manifestó al menos en cuatro etapas:

1) La primera etapa (octubre de 1917-mayo de 1918) comenzó con la escalada rápida de 
algunos sucesos bélicos, ya que con la manifestación de Atomanov, Kaledin y Dutova, se dio pie a la intervención del cuerpo militar de Checoslovaquia, Inglaterra, Francia, América y Japón -actores de la Primera Guerra Mundial-, derivando en la conformación del frente de Oriente.

2) En esos tiempos, los bolcheviques impulsaron la "proletarización" del campo, con la consecuente instauración de la Dictadura del proletariado y del Comité de los pobres, sucesos que desencadenaron la conformación de bases sociales contrarrevolucionarias, que se aliaron temporalmente con el movimiento blanco para dar lugar a la llamada “Contrarrevolución democrática". La ruptura de esta alianza significó el paso a la segunda etapa (diciembre de 1918-junio 1919), misma que se manifestó en una fuerte lucha, que propagó el "terror rojo y blanco".

3) La tercera etapa (julio de 1919-noviembre de 1920) correspondió a la derrota del Ejército blanco, en muchas de las políticas de cambio de los bolcheviques en relación con la clase media campesina.

4) En la cuarta etapa (diciembre de 1920-1922) desaparecieron las secuelas de la Guerra civil, en Oriente, Asia Central y en Transcaucasia, con la derrota de la oposición liberal democrática blanca.

En marzo de 1921 fue declarada la Nueva Política Económica, que consideraba lo siguiente: el cambio de la política de entrega obligatoria de productos agrícolas al Estado; la eliminación de la siembra comunal obligatoria en el campo; la ampliación de las relaciones de mercado; el mantenimiento de la pequeña y mediana empresa; el cambio del trabajo colectivo y del empleo obligatorio; el cambio del pago natural al trabajo -en especie- hacia el pago monetario diferenciado; el cambio hacia la transparencia contable de las grandes corporaciones; la estabilización relativa del sistema financiero; la atracción de inversión extranjera por medio concesiones, etcétera.

Por el contrario, el Estado seguiría manteniendo el control en la esfera bancaria, la propiedad de las grandes y medianas empresas y de los ferrocarriles. Precisamente, esos rasgos pudieron compensar la ausencia del desarrollo de las fuerzas productivas y de una cultura política en la sociedad. Sin embargo, el modelo NEP implicaba varias contradicciones; la principal de ellas era la poca coincidencia de los múltiples sistemas económicos existentes, basados en las formas plurales de propiedad, en el marco del 
régimen autoritario del Partido bolchevique. Estas y otras contradicciones desataron tres crisis: la crisis de sobreproducción, de 1923; la crisis de mercancías, de 1925, y la crisis de abastecimiento y almacenaje de cereales, de 1927 a 1929.

\subsection{Los pilares de un nuevo sistema}

Durante esta época de inestabilidad, la URSS se enfocó en la solución de sus problemas internos. Era requerida la construcción de una sociedad y de una estructura productiva; además, se necesitaba un sistema de cooperación económica, configurado sobre la base de una estructura que favoreciera la industria pesada, la educación y el desarrollo científico y tecnológico.

Por otra parte, la Unión de Repúblicas conformó un Estado unificado, ante la dispersión de las nacionalidades y culturas existentes en su tejido social. Este proyecto fue dirigido por Stalin, durante la época leninista de la URSS e instauró los principios de su modelo personal posterior. Es así que,

en el verano de 1922 surgió la necesidad de dar una nueva forma a las relaciones de la Unión. En el politburó del Comité Central del Partido Comunista, se conformó una comisión encabezada por Stalin, la cual tendría la tarea de desarrollar un proyecto de creación de un estado unificado. Stalin propuso la incorporación de Ucrania, Bielorrusia y Transcaucasia como parte de la Federación Rusa, otorgándoles cierta autonomía para resolver algunos problemas. (Danilov, 2012)

En este contexto, la industrialización soviética es un factor que caracterizó el periodo. Ciertamente, esta tarea no hubiera sido posible sin el apoyo del campo. En un sentido amplio, esta gestión presuponía cambios en el sector agrario, pues era necesario equipar grandes unidades que fueran capaces de aumentar la producción de trigo, carne y otros productos, para abastecer las necesidades de la industria de las ciudades.

Para ello se proponían al menos dos variantes; una de ellas consistía en hacer énfasis en los proveedores más importantes de trigo, quienes, a pesar de las políticas limitativas impuestas por el gobierno, constituían el sector más importante de abastecimiento en el mercado interno. En suma, el camino hacia la construcción de la Rusia socialista condujo al cambio de su postura social. El nuevo paradigma vislumbraba la jerarquía de los obreros y los campesinos pobres sobre la clase social pudiente de la época. 


\section{La génesis de un mundo sin muro}

1989. Era domingo y había una sensación de libertad. Con la desaparición de la URSS, el mundo bipolar se desdibuja; incluso, para algunos -aunque de manera equívoca-, se trataba del fin de la historia. (Fukuyama, 1994) En ese momento, la Guerra Fría, caracterizada por una competencia militar entre las potencias más grandes de la época Estados Unidos y la URSS-, sucumbía ante un dilema económico.

A principios de los años 80, la economía soviética se encontraba en decadencia. La dispersión de los recursos, la falta de infraestructura, la corrupción y el fraude exigían un reordenamiento de la realidad. Sin duda, la mayor falla del modelo económico soviético era la ausencia de estímulos materiales entre la población.

En primer lugar, se pretendía organizar un nuevo modelo económico que permitiera el desarrollo del país. Para lograr este objetivo se llevó a cabo un experimento: se reconstruyó el sistema de transporte y de la industria ligera, y se instauró una forma de contabilidad basada en la transparencia; tomando en cuenta el atraso de la URSS, fue anunciada la aceleración del proceso científico-tecnológico, pero la inversión de capital en esa esfera nunca llegó. En aquel episodio se sufría por una baja calidad de los productos; esta situación provocó la caída de los precios, por lo que el país perdió 20 mil millones de rublos.

Para la recuperación de la calidad de los productos, fue establecido el sistema estatal de supervisión de la producción, situación que instauró el centro de una burocracia mayor, que no generó resultados económicos importantes. Sin embargo, en la población se había dispersado un entusiasmo auténtico por los cambios que se estaban dando; por ello, fue necesario el cambio de los cuadros de gobierno asociados a la nueva estrategia financiera.

Aun así, la reforma consideraba en general los puntos establecidos en el Plan Nacional de Desarrollo, y tomaba en cuenta las condiciones específicas de la ampliación de las empresas, con base en el ideal de autofinanciamiento. Se promovió entonces el renacimiento paulatino del sector privado por medio de la producción cooperativa y se difundió el rechazo al monopolio del comercio exterior, con una mayor integración al mercado internacional.

Asimismo, se promovió la disminución del aparato burocrático y el establecimiento del trabajo conjunto entre los ejidos y las empresas productoras de alimentos, la renta 
cooperativa de la infraestructura agrícola, así como -en la medida de lo posible- la desaparición de las empresas con pérdidas; también se promovió el establecimiento de una red bancaria y con ello la reactivación de la economía. Sin embargo, el permiso para la privatización de las empresas estatales se permitió sólo después de cumplir con las necesidades requeridas por el gobierno, y continuó el control de los precios domésticos por parte del Estado.

De esta manera, con grandes obstáculos, inició la conformación del sector privado. Las inversiones existentes provenían de los mismos miembros de la nomenclatura, que eran los únicos que disponían del capital -muchas veces proveniente de la corrupción y del desvío de recursos nacionales-; por otra parte, se promovió el libre mercado agrícola para igualar los ingresos de los campesinos y de los trabajadores de las ciudades. Más tarde comenzó una reforma en el sistema educativo, que buscaba otorgar autonomía a las instituciones.

El primer paso de esta reforma económica -conocida como Perestroika-, fue la reconstrucción del sistema político; por tanto, de los 460 miembros y candidatos del Comité Central, fueron excluidos rápidamente 110. Fue entonces cuando Gorbachov se convirtió en el primer presidente de la Unión Soviética.

Posteriormente comenzó la ruptura de la estructura soviética. Se declaró la independencia de las repúblicas bálticas; en primer lugar, Ucrania y Transcaucasia. Asimismo, en este mismo periodo, desapareció el Consejo de Ayuda Mutua Económica (CAME), y con ello toda forma de abastecimiento de productos agrícolas y de consumo; lo cual propició una rearticulación de las cadenas productivas internas. En consecuencia, la cooperación que hasta entonces se conservaba con los aliados, se vino abajo.

Por otra parte, la democratización de la vida social no dejó de reflejarse en la esfera de las relaciones regionales. Los problemas que se habían acumulado por años y que el gobierno ignoraba, se expresaron de repente con el espíritu de las libertades iniciales. Una de las primeras manifestaciones se concentró en el desacuerdo por la creciente disminución de la cantidad de escuelas con programas de estudio impartidos en el idioma local, haciendo a un lado el uso del idioma ruso. En tanto, se realizaban grandes manifestaciones en las repúblicas transcaucásicas que exigían la sustitución de funcionarios rusos por locales. Las repúblicas bálticas se manifestaron en contra del 
idioma ruso como idioma oficial y proclamaron la oficialización de su propia lengua, oficializando así su separación de la URSS.

El mismo proceso se generalizó entre las repúblicas restantes ${ }^{2}$ y por primera vez en muchos años apareció el peligro de la proliferación del fundamentalismo islámico, lo que puede explicar el riesgo de vínculos con el terrorismo, surgido posteriormente en los países del Medio Oriente.

Al inicio, la llegada al poder de Gorbachov no vaticinaba ningún cambio en particular. Fue proclamada la necesidad de un sistema de defensa militar y de la permanencia del mismo ritmo de fortalecimiento de los lazos de amistad con los países socialistas, así como el apoyo a los movimientos similares en otros países. En realidad, la política exterior de la URSS comenzó a notarse con el nombramiento de Shevardnadze como ministro de relaciones exteriores, en 1985. Entonces fueron definidas las principales acciones de la política exterior: normalización de las relaciones con los países de Occidente -en primer lugar, con los Estados Unidos-, la disminución bilateral de la producción de armamento, y la desaparición de un potencial enfrentamiento militar con Norteamérica y sus aliados en Asia, África y América Latina.

En el mismo año se celebró la primera reunión entre Gorbachov y Reagan, misma que inauguró el acercamiento de los vínculos entre ambos hemisferios. En 1987, la URSS y los EE. UU. firmaron el acuerdo sobre la destrucción de un tipo de armas nucleares misiles de corto y mediano alcance- que constituían un peligro particular para los aliados europeos de Norteamérica.

Entre 1988 y 1989, los principios ideológicos influían cada vez menos en la política de Gorbachov, sobreponiéndose los económicos, acompañados de un pragmatismo que poco a poco se posicionó en el ideario de gobierno para la toma de decisiones. El empeoramiento de las condiciones económicas en la URSS propició una influencia cada vez más importante de Occidente. En la reunión de Malta de 1989, Gorbachov anunció la "Muerte de la Doctrina Brershnev", lo cual permitió a George Bush fortalecer las acciones en contra del socialismo. De manera casi unilateral, la Unión disminuyó en un $40 \%$ la posesión de sus armas más potentes.

\footnotetext{
${ }^{2}$ Georgia, Lituania, Estonia, Letonia, la Federación de Rusia, Uzbekistán, Moldavia, Ucrania y Bielorrusia declararon su independencia de la URSS en 1990.
} 
Por su parte, en verano de 1991, el entonces presidente de EE. UU. declaró seis condiciones para una mayor cooperación con la URSS: democracia, mercado, federalismo, rechazo a la modernización en la producción de misiles nucleares y cambio de la política hacia el Medio Oriente y África. Por primera vez en 70 años, los Estados Unidos no sólo imponían medidas sobre la resolución de los problemas internacionales, sino que se inmiscuían en los asuntos internos de la Unión Soviética. Paralelamente influían en las repúblicas de la Unión a favor de Gorbachov.

En verano del 90, Gorbachov autorizó la unificación de Alemania y su permanencia en la Organización del Tratado del Atlántico Norte (OTAN). Él pensaba que, por ser accesible ante los deseos de su contraparte, podría fortalecer su propia posición en la URSS; pero la desaparición del Pacto de Varsovia y del bloque socialista impactaron aún más en los intereses soviéticos. Por tanto, que se incrementaron las críticas a su administración, causando que el sistema socialista dejara de existir, sin ningún disparo.

El cambio de la ideología en la URSS permitió mejorar sus relaciones con Sudáfrica, Corea del Sur, Taiwán e Israel. Asimismo, el "nuevo pensamiento" tuvo resultados contradictorios. Por una parte, su gran logro no sólo fue el debilitamiento de la amenaza de la guerra nuclear en Oriente y Occidente, sino que también se comenzó a hablar del fin de la Guerra Fría. Esta nueva realidad propició una situación internacional que permitió hablar de EE. UU. como único ganador de esa guerra. La URSS y el bloque socialista fueron vencidos y dejaron de existir. Esto condujo a la caída del sistema bipolar de las relaciones internacionales, simbolizado por la destrucción del Muro de Berlín.

El poder de cambiar el mundo, no es un asunto menor. La revolución de 1917, que dio lugar al surgimiento de la URSS y a la destrucción del Muro de Berlín -como símbolo del fin de la era soviética- son dos momentos históricos que, sin duda, sembraron en el imaginario colectivo una pauta para la reflexión continua. Por tanto, Rusia tuvo un papel fundamental en la construcción de un mundo diferente.

\section{Bibliografía}

Danilov, A. (2012). Historia de Rusia. Moscú.

Fukuyama, F. (1994). El fin de la historia y el último hombre. México: Planeta. 
Lapshov, G. I. y Sheremitiev, K. (1990). México: Capitalismo y Sociedad, Contradicciones de su Desarrollo. Moscú: Nauka.

Rutherford, E. (1991). Rusia. México: Roca Editorial. 